\title{
Time-dependent effects in melting and phase change for laser-shocked iron
}

\author{
S. White, ${ }^{1}$ B. Kettle, ${ }^{1}$ J. Vorberger, ${ }^{2}$ C. L. S. Lewis, ${ }^{1}$ S. H. Glenzer, ${ }^{3}$ E. Gamboa, ${ }^{3}$ B. Nagler, ${ }^{3}$ F. Tavella, ${ }^{3}$ H. J. Lee, ${ }^{3}$ \\ C. D. Murphy, ${ }^{4}$ D. O. Gericke, ${ }^{5}$ and D. Riley ${ }^{1}$ \\ ${ }^{1}$ Centre for Plasma Physics, School of Mathematics and Physics, Queen's University Belfast, University Road, \\ Belfast BT7 1NN, United Kingdom \\ ${ }^{2}$ Helmholtz-Zentrum Dresden-Rossendorf, 01328 Dresden, Germany \\ ${ }^{3}$ SLAC National Accelerator Laboratory, Menlo Park, California 94025, USA \\ ${ }^{4}$ Department of Physics, University of York, Heslington, York YO10 5DD, United Kingdom \\ ${ }^{5}$ Centre for Fusion, Space and Astrophysics, Department of Physics, University of Warwick, Coventry CV4 7AL, United Kingdom
}

(Received 30 September 2019; revised 15 January 2020; accepted 31 July 2020; published 3 September 2020)

\begin{abstract}
Using the Linac Coherent Light Source facility at the Stanford Linac Coherent Light Source National Accelerator Laboratory, we have observed x-ray scattering from iron compressed with laser-driven shocks to earth-core-like pressures above $400 \mathrm{GPa}$. The data show cases where melting is incomplete and we observe hexagonal-close-packed crystal structure at shock compressed densities up to $14.0 \mathrm{~g} \mathrm{~cm}^{-3}$ but no evidence of a double-hexagonal-close-packed crystal. The observation of a crystalline structure at these densities, where shock heating is expected to be in excess of the equilibrium melt temperature, may indicate superheating of the solid. These results are important for equation of state modeling at high strain rates relevant for impact scenarios and laser-driven shock-wave experiments.
\end{abstract}

DOI: 10.1103/PhysRevResearch.2.033366

\section{INTRODUCTION}

Iron is the stable product of nuclear burn in massive stars and thus highly abundant on many planets in and outside our solar system. The earth's core and mantle are of course the best studied cases of iron dominated by its high-pressure properties. The position of the melting transition between the solid inner core and the liquid outer core determines the inner structure of the planet and the generation of its magnetic field and also restricts the abundance of light elements in the core. To reach these extreme conditions, shock-wave experiments are commonly applied, often with optical diagnostics. Here we report on such an experiment but additionally we have observed $\mathrm{x}$-ray scattering from the sample shortly after the shock wave created pressures in excess of $400 \mathrm{GPa}$. The data include cases of incomplete melting and we observe a hexagonal-close-packed (hcp) crystal structure at densities up to $14 \mathrm{~g} \mathrm{~cm}^{-3}$, but no evidence of the predicted doublehexagonal-close-packed (dhcp) structure. As shock heating is in excess of the equilibrium melt temperature, these data demonstrate important timescale effects during the phase changes: We propose superheating of the solid and a slow transition into a new lattice as an explanation. These results have important implications for the interpretation of experiments and modeling of matter at high strain rates. Our

Published by the American Physical Society under the terms of the Creative Commons Attribution 4.0 International license. Further distribution of this work must maintain attribution to the author(s) and the published article's title, journal citation, and DOI. experiments show that even at nanosecond timescales, both solid-solid and melting phase changes do not occur as indicated in equilibrium equations of state tables. Thus, typical laser-driven shock-wave experiments might indicate wrong structural and thermodynamic results when being interpreted under the equilibrium assumption. Moreover, the modeling of impact events must also consider the dynamics of phase changes.

It is widely understood that the extreme pressures and temperatures in planetary cores $\left(P>100 \mathrm{GPa}\right.$ and $T>10^{4} \mathrm{~K}$ for earth) create complex states characterized by strongly interacting particles, partially degenerate electrons, and partial (unknown) ionization [1-5]. Understanding the thermodynamic properties of such matter requires detailed knowledge of its structural state. Modeling these properties presents significant theoretical challenges in particular close to phase boundaries. Experimentally, the required high pressures can often only be reached dynamically, which raises the question of equilibration times and sufficiently stable states for probing.

Due to high abundance in the cores of earth and earthlike planets, iron is of particular interest in geosciences and both static compression and shock-wave experiments have been used to explore the equation of state and melting at high pressure [6-18]. It is expected that shock melting begins at about $220 \mathrm{GPa}$ and is complete by $280 \mathrm{GPa}[8,9]$. A phase change from bcc to hcp occurs in both static and shock compression at a relatively modest pressure of $\sim 13 \mathrm{GPa}$ [10-12]. Further phase changes have been discussed for both shock [13,14] and static compression $[15,16]$. In particular, a transition from hcp to dhcp has been reported [16,17], as well as stability of the bcc structure at earth core conditions [18] and stability of the 
$\gamma$ (fcc) phase for highly compressed iron $[19,20]$. As these phase changes are predicted to occur at extreme conditions, intense nanosecond duration lasers are commonly used to reach the required shock pressures. We have carried out such an experiment and added x-ray diffraction as a diagnostic to directly observe the microscopic structure of the states created. Our data highlight the fact that phase changes may not be completed even on a nanosecond timescale and that the dynamics of the process may be of great importance for the interpretation of experimental data from shock-compressed matter.

\section{EXPERIMENTAL METHOD}

In this work, our x-ray-scattering measurements were taken on samples of warm dense iron created using laserdriven shock compression at the Matter in Extreme Conditions (MEC) end station of the Linac Coherent Light Source (LCLS) x-ray free-electron laser $[21,22]$. The target samples were polycrystalline iron foils of $10.2 \pm 0.3 \mu \mathrm{m}$ thickness and coated with $5.0 \pm 0.1 \mu \mathrm{m}$ of $\mathrm{CH}$ on one side. Ion beam milling of foil samples followed by scanning electron microscopy of the sample cross section indicates micron-sized crystallites with no significant porosity. The two optical laser beams of the MEC end station $(527 \mathrm{~nm})$ were focused with the use of random phase plates to a focal spot of either 100or 50- $\mu \mathrm{m}$ nominal diameter onto the $\mathrm{CH}$-coated side of the samples (See Fig. 1). The optical pulse shape rose in about 0.5 ns with a FWHM of 1.6 ns peaking at an intensity of $2 \times 10^{13} \mathrm{~W} \mathrm{~cm}^{-2}(100-\mu \mathrm{m}$ spot shot) before falling off over $0.5 \mathrm{~ns}$. The LCLS beam was focused with a Be lens to a spot of $20 \mu \mathrm{m}$ diameter and centered on the optical focal spot. Monitoring of the overlap during the experiment on several test shots (see [23]) indicated that the centers of the optical and $\mathrm{x}$-ray spot were coincident to better than $20 \mu \mathrm{m}$.

A velocity interferometer system for any reflector (VISAR) monitored the shock breakout from the rear of the samples. Figure 1(c) shows a typical VISAR data image. Approximately $20 \%$ of the laser energy is diffracted by the phase plate, outside the central spot region to form a lower intensity pedestal. Coupled with refraction in the $\mathrm{CH}$ plasma and some lateral shock spreading, this causes the affected region to be a little wider than the main $100-\mu \mathrm{m}$ focal spot size. The shock breakout was accompanied by a sudden drop in reflectivity associated with the heating and decompression of the rear surface, which produces a highly absorbing plasma layer. The principal diagnostic of the scattered $\mathrm{x}$ rays was the Cornell-SLAC hybrid pixel array detector (CSPAD) $560 \mathrm{~K}$ [24].

\section{RESULTS}

Figure 2 shows raw data images from the CSPAD for two shots taken with the $100-\mu \mathrm{m}$ focal spot. We start with this case because the ratio of the focal spot size to target thickness allows meaningful comparison with a one-dimensional hydrodynamic code to estimate expected shock velocity and probed density. The smooth bcc diffraction lines seen in Fig. 2(a) are in fact also present in Fig. 2(b) but are less visible and are in all shots. In situ testing confirmed that this
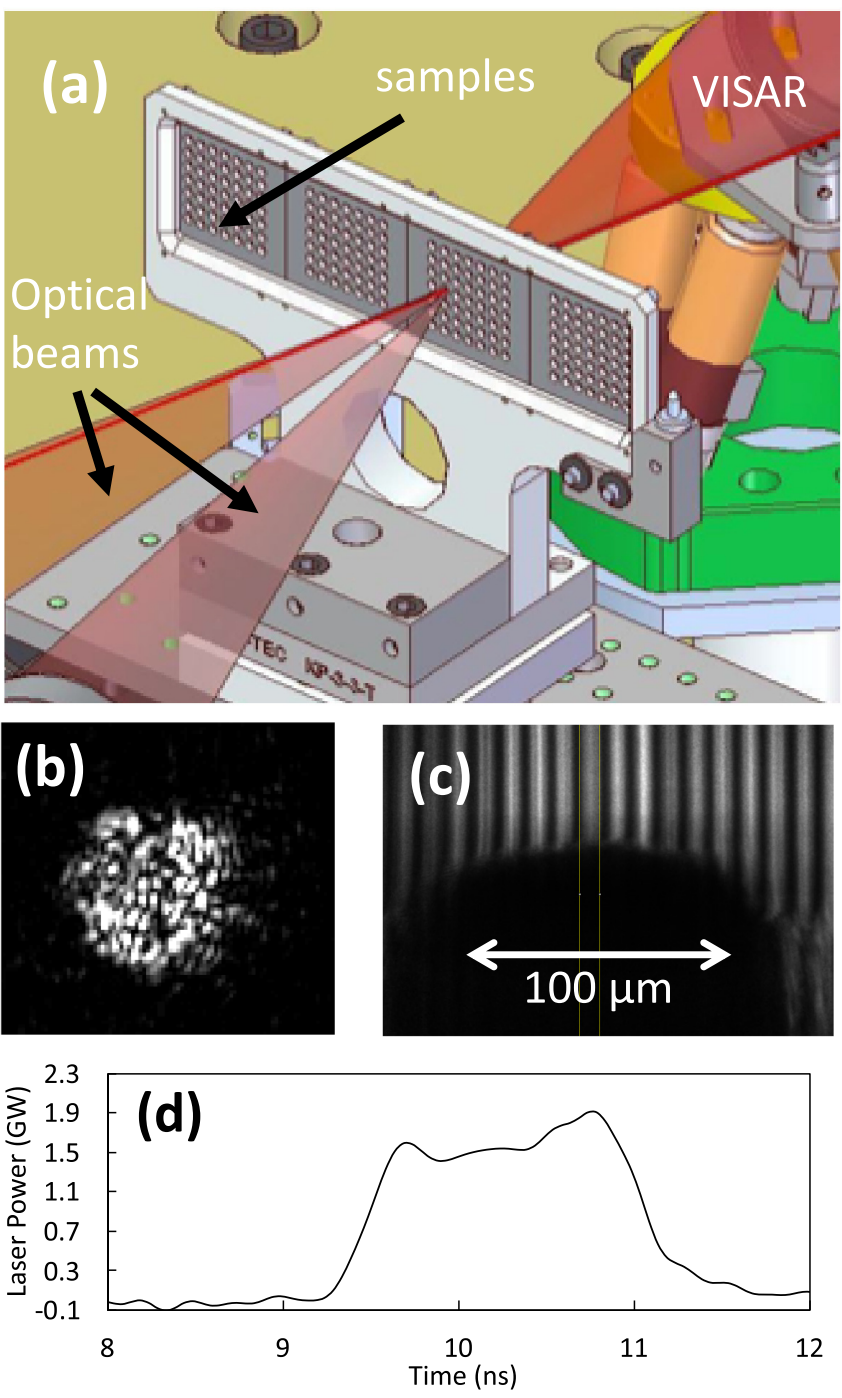

FIG. 1. (a) Schematic of the experimental arrangement. The LCLS beam (solid red line) passes through the samples at an angle of $24^{\circ}$ to the target normal with a $20-\mu \mathrm{m}$ diameter. The optical beams are incident on the target at an angle of $16^{\circ}$ on either side of the target normal. (b) Focal spot image of the beams with the $100-\mu \mathrm{m}$ phase plate. (c) VISAR data showing breakout when using the nominal $100-\mu \mathrm{m}$ phase plate. The central $100-\mu \mathrm{m}$ region is overall relatively flat (see the text for a discussion of the width of the shocked region). (d) Oscilloscope trace of the optical pulse shape.

is caused as the beryllium lens scatters a small portion of the beam $(\sim 1 \%)$ into a millimeter-sized region of cold foil surrounding the main focal spot. In Fig. 2(b) we also note brighter, spotty bcc features that are seen when we probe just ahead of shock breakout and there is a small amount of unshocked iron ahead of the main x-ray beam. These features serve as additional calibration of our angle scale on each shot.

We see complete melting in Fig. 2(a) with the expected liquid-type diffraction feature which can be described with density functional theory (DFT) molecular dynamics (MD). In Fig. 2(b) complete melting has not occurred and we see strong evidence that a significant portion of the probed volume is in 

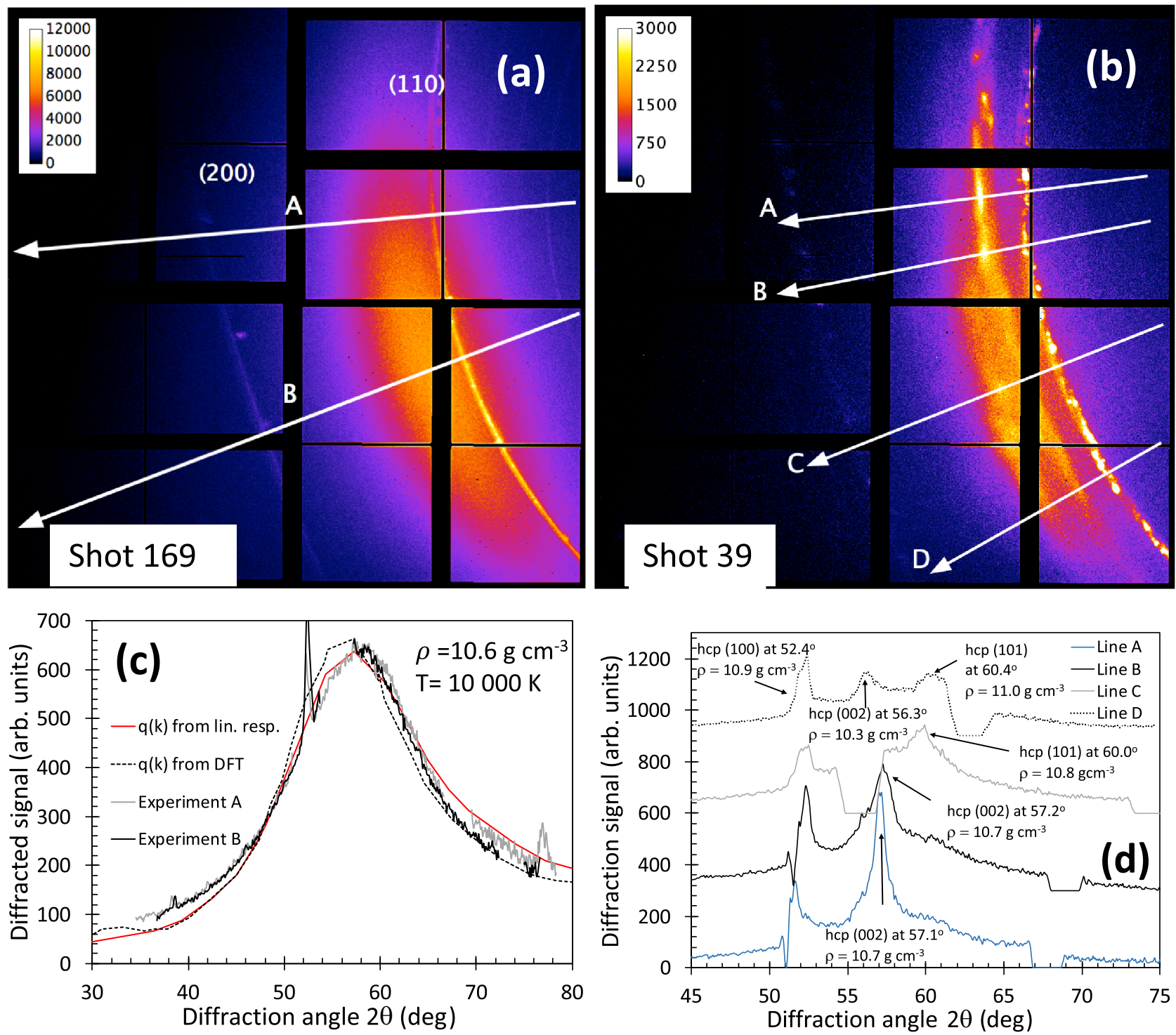

FIG. 2. (a) Raw diffraction data for shock melted iron. The probe time was $\sim 40$ ps before shock breakout. (b) Data for a shot similar to that in (a) with a probe time of $170 \mathrm{ps}$ ahead of shock breakout. The labeled arrows indicate lineouts shown below. (c) Two lineouts of the melted shot in (a) showing good consistency as a check on the image processing. The data are fitted with a DFT simulation with the electron-ion correlation term calculated in two ways as described in the text. (d) Lineouts indicated in (b). The hcp (001) features are closely coincident with the bcc (110) reflection.

the hcp crystalline phase. The densities assigned to individual hcp diffraction features range from 10.3 to $11.0 \mathrm{~g} \mathrm{~cm}^{-3}$. Figure 2(c) shows a lineout of the liquid diffraction feature from Fig. 2(a). We have modeled the data using ab initio DFT MD simulations. The structure may be understood as that of a strongly coupled one-component plasma with ion charge state $Z=8$ and degenerate Yukawa-type screening of the ion-ion interaction (see the Supplemental Material [23]). Fitting to the experimental data gives a best fit to the average density of $(10.6 \pm 0.3) \mathrm{g} \mathrm{cm}^{-3}$ with a temperature of $10^{4} \pm 0.2 \times$ $10^{4} \mathrm{~K}$. The error bars are estimated by performing leastsquares fits to a series of DFT MD simulations, adjusting the scaling constant between experiment and simulation in each shot to minimize the least-squares sum. This is similar to the density range seen from the crystalline features in Fig. 2(b). In Fig. 2(d) we see lineouts of the unmelted case. The hcp (001) feature is closely coincident with the bcc (110) in this case but is distinct from it in other data shots. Analysis of both VISAR channels (discussed below), including camera jitter, indicates that for the fully melted case (shot 169) we have breakout at $1.04 \pm 0.04 \mathrm{~ns}$. The probe time was set to $1.0 \mathrm{~ns}$. For the incompletely melted case (shot 39 ), breakout was similarly estimated as $1.07 \pm 0.04 \mathrm{~ns}$ and probing was at $0.9 \mathrm{~ns}$. In Fig. 3 we see average intensities for the fringes for the VISAR data of Fig. 2, illustrating how there is little discernible difference in the shock breakout time for the two cases.

For data taken with $50-\mu \mathrm{m}$ focal spots, we expect stronger nonplanar behavior, mostly as a result of the much longer 


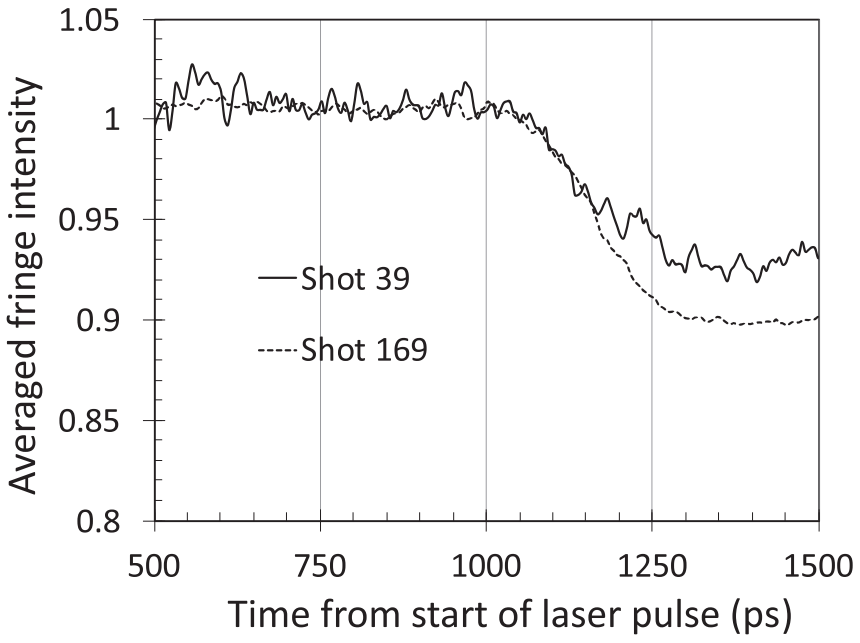

FIG. 3. Comparison of the average fringe intensity from the VISAR diagnostic for the two shots shown in Fig. 2. There is little discernible difference in the breakout time recorded for these two shots.

scale length of the $\mathrm{CH}$ plasma but also the smaller ratio of spot size to target thickness. However, as seen in Fig. 4, we were able to collect clear data showing higher density for shots both with melted and with crystalline iron. For unmelted or partially melted iron we see the hcp phase with features indicating densities between 12.7 and $14.0 \mathrm{~g} \mathrm{~cm}^{-3}$ and the melted shot can be fitted using DFT MD to a density of $(13.0 \pm 0.5) \mathrm{g} \mathrm{cm}^{-3}$. The temperature best fit is $1.5_{-0.1}^{+0.3} \times$ $10^{4} \mathrm{~K}$. The unmelted shot is for early probing $0.6 \mathrm{~ns}$ before shock breakout. Similar data for probing closer to shock breakout are shown in the Supplemental Material [23]. Our fits and error bars, in the liquid case, refer to the estimate of the average density; stronger lateral gradients mean that there is evidence of a wider range of densities than for the larger focal spot data.

\section{HYDRODYNAMIC SIMULATION}

We have performed a hydrodynamic simulation of shot 169 [Fig. 5(a)] with the HYADES code [25] using the SESAME equation of state 2140 for iron [26]. At the probe time, the simulated shock front has a density $\sim 13.5 \mathrm{~g} \mathrm{~cm}^{-3}$ and velocity of $11.5 \mathrm{~km} / \mathrm{s}$ at the time of breakout. Although we expect to be probing off-Hugoniot states, as a point of reference, we note that, for a 400-GPa shock, the Hugoniot from the SESAME equation of state used agrees with published Hugoniot data [13] to better than $2 \%$ in shock velocity and better than $4 \%$ in density. The simulated averaged density in the iron is $11.7 \mathrm{~g} \mathrm{~cm}^{-3}$, about $10 \%$ higher than our DFT fit, which may be due to some small lateral shock spreading. Although there is variation in compressed density through the Fe sample, the simulation predicts that most of the target is within $\pm 10 \%$ of the average density. The simulated shock front temperature is $1.3 \mathrm{eV}(15000 \mathrm{~K})$, which is higher than our DFT fit. However, both temperatures are well above the expected equilibrium melt temperature for this pressure range [27-29]. The simulated temperature is higher behind the shock front as the shock at this point is decaying from a peak simulated pressure close to $10 \mathrm{Mbar}$ which occurs as the shock enters the iron at around $400 \mathrm{ps}$ after the start of the optical laser pulse. This is an important point as we probe the whole volume of the iron and due to the decaying shock expect our conditions at the time of probing to be off the Hugoniot away from the region where the equation of state is usually tested against experiment.

In Fig. 5(b) we show a plot in density-pressure space of the states we access in the data shown above. The density and temperature from the DFT fits gives us the pressure. As we can see, we are probing states at pressures above the Hugoniot for the given density. This is expected from the hydrodynamic simulation where the shock has decayed from much higher pressures at the time of probing, leaving a higher temperature behind the shock front.

\section{DISCUSSION}

As we have seen above, we see a mixture of melting in some cases and nonmelting in others, despite no obvious differences in irradiation conditions between shots. The identification of the crystalline features means that we assign a range of densities to the features that is broadly consistent with both the liquid cases and simulation where this is appropriate. Other identifications of the diffraction features do not allow this and hcp is a known phase of compressed iron. It is known that the hcp phase can be generated rapidly as discussed, for example, by Hawreliak et al. [30], who reported it to be on a picosecond timescale, much shorter than the approximately 0.5 -ns rise time of our laser. In fact, in previous work on nanosecond shock compression of iron at pressures up to $170 \mathrm{GPa}$, the hcp phase was observed in x-ray-diffraction measurements [31].

A possible explanation for our data may be the known phenomenon of superheating [32-37], in which the probability of melting nucleation in the small volume shocked depends on a normalized energy barrier. The degree to which a solid may be superheated above the equilibrium melting temperature [34] is a function of the energy barrier required as well as a weak function of the heating rate. Luo and Ahrens [34] showed that, for iron, a maximum superheating of around $25 \%$ above the equilibrium melt temperature is expected at our heating rates of $\sim 10^{13} \mathrm{~K} \mathrm{~s}^{-1}$. This result can be obtained using thermodynamic parameters for iron under ambient conditions, but Luo and Ahrens gave arguments to show that the result should hold for our pressure regime [37]. Thus, we can expect to be able to reach $\sim 9000 \mathrm{~K}$ before melting, based on an equilibrium melt temperature of $\sim 7000 \mathrm{~K}$ for our shock compressed pressure [27-29]. This is broadly consistent with the temperature seen in the DFT fits to the melted shot data for Fig. 2. Evidence of superheating has been reported previously for shocked Fe [37] and other materials [32], based on optical emission data.

It is not possible to have a direct measure of the temperature from the crystalline hcp features under these conditions. However, for the data in Fig. 2, the shock breakout times and densities observed are consistent between the melted and partially melted cases and both are consistent with the simulated values where shock speeds in $\mathrm{Fe}$ are above $10 \mathrm{~km} \mathrm{~s}^{-1}$ and thus consistent with pressures above $300 \mathrm{GPa}$. 

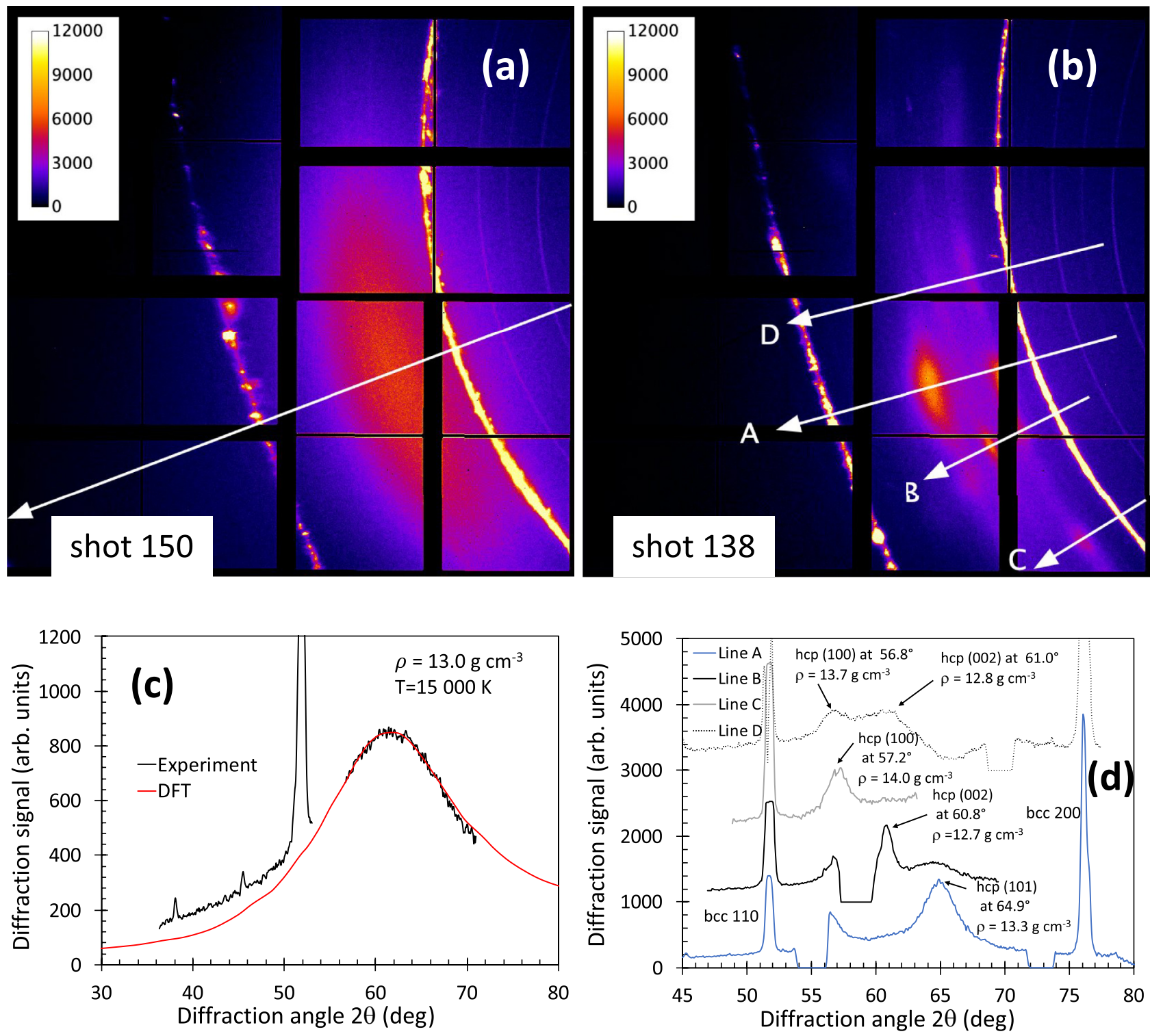

FIG. 4. Data for the 50- $\mu \mathrm{m}$ optical focal spot. (a) Shock breakout is $1.0 \mathrm{~ns}$ and probing at $0.8 \mathrm{~ns}$. We see a liquid Fe sample that can be fitted to $13 \mathrm{~g} \mathrm{~cm}^{-3}$. (b) The shock breakout is $1.1 \mathrm{~ns}$ and probing is at $0.5 \mathrm{~ns}$. There are clear hcp diffraction features noted. The Bragg positions of the various features indicate density ranging from 12.7 to $14.0 \mathrm{~g} \mathrm{~cm}^{-3}$ with a mean and standard deviation of $13.4 \pm 0.5 \mathrm{~g} \mathrm{~cm}^{-3}$. We can see that there appears to be three distinct groups of features. (c) DFT simulation of the data in (a) showing a fit to $13.0 \mathrm{~g} \mathrm{~cm}^{-3}$ and $1.5 \times 10^{4} \mathrm{~K}$. The fit is not as close as for the lower density shot. This is most likely due to the smaller focal spot, meaning that nonuniformity in the conditions probed start to become more significant. (d) Lineouts as indicated in (b). We can see several hcp features identified along with angle and inferred density. The range of densities is compatible with the expected range from simulation. The faint diffraction features at lower angles are due to the third-order LCLS beam at $21 \mathrm{keV}$.

Furthermore, the densities seen in Fig. 4 are above the expected density of $12.6 \mathrm{~g} \mathrm{~cm}^{-3}$, for shock melting on the Hugoniot [13]. Since the shock heating expected along the Hugoniot rises more rapidly with pressure than the equilibrium melt temperature, it is clear that we are well above the equilibrium melt temperature, since we are able to observe shock melting at the lower-intensity shock drive of the first set of data in Fig. 2.

In this work we have direct structural evidence from the bulk of the sample that shows us not only that there is a crystalline structure but that it is hcp, which is an expected phase change for relatively low shock pressure. We do not see evidence for dhcp, which has been reported as a solidsolid phase change $[16,17]$ closer to $200 \mathrm{GPa}$. Rethfeld et al. [38] calculated the homogeneous nucleation time for different materials for iron under ambient conditions. These data allow us to estimate that the nucleation time, for $25 \%$ superheating, is well above the nanosecond level, but there is a rapid dependence on the level of heating. We note that our experiment was carried out with polycrystalline samples and that future work 

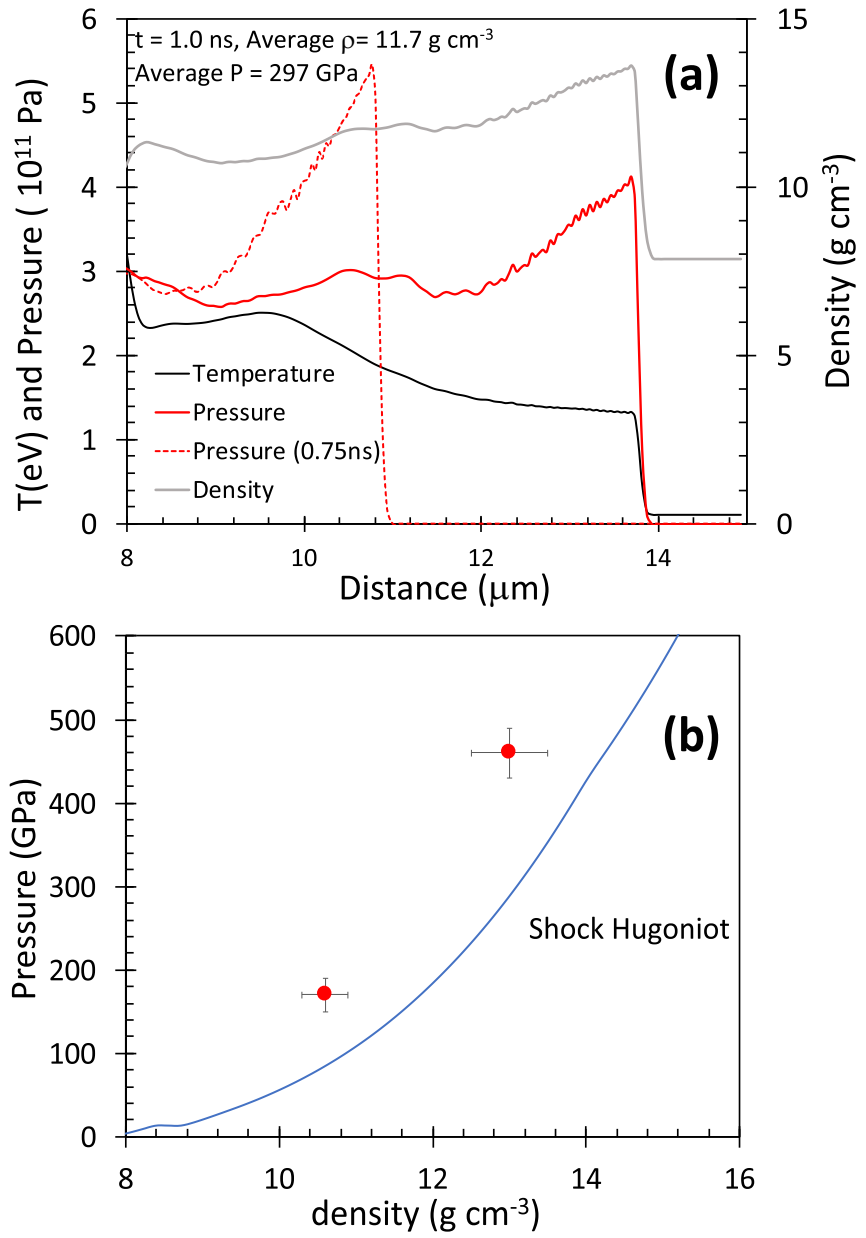

FIG. 5. Hydrodynamic simulation of shot 169 using the HYADES radiation-hydrodynamics code using the measured pulse shape and energy. The code uses multigroup diffusion for radiation transport in 35 groups logarithmically spaced from 0.01 to $15 \mathrm{keV}$. The ionization model is Thomas-Fermi and the SESAME equation of state 2140 for Fe is used. We have also plotted the pressure profile at an earlier time $(0.75 \mathrm{~ns})$ to illustrate the fact that the shock pressure is decaying and that the whole of the target is subjected to shock pressure and temperature well in excess of what is expected to be required for melting. In the figure, we have only included the Fe layer as that is by far dominant in the scattering. (b) Relative positions of our data compared to the shock Hugoniot. The decaying shock means we are probing the measured densities at temperatures in excess of the equivalent shock temperature on the Hugoniot. should investigate the role of the crystallite boundaries in nucleation by comparing data to data taken with single crystals.

\section{CONCLUSION}

In summary, we have observed $\mathrm{x}$-ray scattering from iron under shock compressed conditions where completion of melting would be expected. The data have two significant outcomes. First, it suggests that superheating might be a key phenomenon in our experiment and we have observed this, not through observing optical emission and inferring a temperature as in previous work [32,36], but by direct observation of the shock compressed crystal structure. Second, we have seen no evidence of dhcp or another solid crystalline phase beyond the bcc-hcp phase change. Such a further phase change has been reported by others for static compressions and for longer timescales in shock experiments. Our results indicate that there is more work that can be done to explore the implementation of equations of state for hydrodynamic modeling of laser-driven shocks and high-pressure impact scenarios on nanosecond timescales. This may mean that the application of nansosecond-duration shock drive techniques in attempting to reproduce conditions of relevance for planetary and geosciences may, depending on the particular case, need careful consideration.

Supplemental data are available via [39].

\section{ACKNOWLEDGMENTS}

This work was supported by UK Engineering and Physical Sciences Research Council Grant No. EP/K009591/1. Use of the LCLS, SLAC National Accelerator Laboratory, was supported by the U.S. Department of Energy, Office of Science, Office of Basic Energy Sciences under Contract No. DE-AC02-76SF00515 and Fusion Energy Sciences FWP 100182. The MEC instrument was supported by the U.S. Department of Energy, Office of Science, Office of Fusion Energy Sciences under Contract No. SF00515. The DFT computations were performed on a Bull Cluster at the Center for Information Services and High Performance Computing (ZIH) at TU Dresden. We would like to thank the ZIH for its support and generous allocations of computer time.
[1] A. H. Cook, Interiors of the Planets (Cambridge University Press, Cambridge, 1980).

[2] Treatise on Geophysics, Volume 10: Planets and Moons, edited by T. Spohn (Elsevier, Amsterdam, 2007), Vol. 10

[3] R. W. Lee et al., J. Opt. Soc. Am. B 20, 770 (2003).

[4] A. Ng, T. Ao, F. Perrot, M. W. C. Dharma-Wardana, and M. E. Foord, Laser Part. Beams 23, 527 (2005).

[5] M. Koenig et al., Plasma Phys. Control. Fusion 47, B441 (2005).

[6] R. Boehler, Nature (London) 363, 534 (1993).
[7] Q. Williams, R. Jeanloz, J. Bass, B. Svendsen, and T. J. Ahrens, Science 236, 181 (1987).

[8] J. H. Nguyen and N. C. Holmes, Nature (London) 427, 339 (2004).

[9] J. M. Brown and R. G. McQueen, J. Geophys. Res.-Solid Earth 91, 7485 (1986).

[10] D. Bancroft, E. L. Peterson, and S. Minshall, J. Appl. Phys. f27, 291 (1956).

[11] J. C. Jamieson and A. W. Lawson, J. Appl. Phys. 33, 776 (1962).

[12] D. H. Kalantar, J. F. Belak, G. W. Collins, J. D. Colvin, H. M. Davies, J. H. Eggert, T. C. Germann, J. Hawreliak, B. L. Holian, 
K. Kadau, P. S. Lomdahl, H. E. Lorenzana, M. A. Meyers, K. Rosolankova, M. S. Schneider, J. Sheppard, J. S. Stölken, and J. S. Wark, Phys. Rev. Lett. 95, 075502 (2005).

[13] J. M. Brown, J. N. Fritz, and R. S. Hixson, J. Appl. Phys. 88, 5496 (2000).

[14] J. M. Brown, Geophys. Res. Lett. 28, 4339 (2001).

[15] L. S. Dubrovinsky, S. K. Saxena, F. Tutti, S. Rekhi, and T. LeBehan, Phys. Rev. Lett. 84, 1720 (2000).

[16] C. S. Yoo, J. Akella, A. J. Campbell, H. K. Mao, and R. J. Hemley, Science 270, 1473 (1995).

[17] L. Vocadlo, J. Brodholt, D. Alfe, G. D. Price, and M. J. Gillan, Geophys. Res. Lett. 26, 1231 (1999).

[18] A. B. Belonoshko, T. Lukinov, J. Fu, J. J. Zhao, S. David, and S. I. Simak, Nat. Geosci. 10, 312 (2017).

[19] L. Stixrude, Phys. Rev. Lett. 108, 055505 (2012).

[20] C. J. Pickard and R. J. Needs, J. Phys.: Condens. Matter 21, 452205 (2009).

[21] S. H. Glenzer et al., J. Phys. B 49, 092001 (2016).

[22] B. Nagler et al., J. Synchrotron Radiat. 22, 520 (2015).

[23] See Supplemental Material at http://link.aps.org/supplemental/ 10.1103/PhysRevResearch.2.033366 for details.

[24] S. Herrmann et al., Nucl. Instrum. Methods Phys. Res. Sect. A 718, 550 (2013).

[25] J. T. Larsen and S. M. Lane, J. Quant. Spectrosc. Radiat. Transfer 51, 179 (1994).
[26] S. P. Lyon, and J. D. Johnson, Los Alamos National Laboratory Report No. LA-UR-92-3407, 1992 (unpublished).

[27] S. Anzellini, A. Dewaele, M. Mezouar, P. Loubeyre, and G. Morard, Science 340, 464 (2013).

[28] J. Bouchet, S. Mazevet, G. Morard, F. Guyot, and R. Musella, Phys. Rev. B 87, 094102 (2013).

[29] A. B. Medvedev, Combust. Explo. Shock 50, 582 (2014).

[30] J. Hawreliak et al., Phys. Rev. B 74, 184107 (2006).

[31] A. Denoeud et al., Proc. Natl. Acad. Sci. USA 113, 7745 (2016).

[32] D. A. Boness and J. M. Brown, Phys. Rev. Lett. 71, 2931 (1993).

[33] Q. S. Mei and K. Lu, Prog. Mater. Sci. 52, 1175 (2007).

[34] S.-N. Luo and T. J. Ahrens, Appl. Phys. Lett. 82, 1836 (2003).

[35] S.-N. Luo, T. J. Ahrens, T. Çağin, A. Strachan, W. A. Goddard III, and D. C. Swift, Phys. Rev. B 68, 134206 (2003).

[36] M. Millot, N. Dubrovinskaia, A. Černok, S. Blaha, L. Dubrovinsky, D. G. Braun, P. M. Celliers, G. W. Collins, J. H. Eggert, and R. Jeanloz, Science 347, 418 (2015).

[37] S.-N. Luo and T. J. Ahrens, Phys. Earth Planet. Inter. 143-144, 369 (2004).

[38] B. Rethfeld, K. Sokolowski-Tinten, D. von der Linde, and S. I. Anisimov, Phys. Rev. B 65, 092103 (2002).

[39] https://pure.qub.ac.uk/. 